\title{
A retrospective study of neonatal case management and outcomes in rural Rwanda post implementation of a national neonatal care package for sick and small infants
}

Merab Nyishime ${ }^{1 *}$ (D), Ryan Borg ${ }^{1}$, Willy Ingabire ${ }^{1}$, Bethany Hedt-Gauthier ${ }^{1,3}$, Evrard Nahimana ${ }^{1}$, Neil Gupta ${ }^{1,4}$, Anne Hansen ${ }^{5}$, Michelle Labrecque ${ }^{5}$, Fulgence Nkikabahizi², Christine Mutaganzwa', Francois Biziyaremye', Claudine Mukayiranga ${ }^{2}$, Francine Mwamini ${ }^{2}$ and Hema Magge ${ }^{1,4,5,6}$

\begin{abstract}
Background: Despite worldwide efforts to reduce neonatal mortality, $44 \%$ of under-five deaths occur in the first 28 days of life. The primary causes of neonatal death are preventable or treatable. This study describes the presentation, management and outcomes of hospitalized newborns admitted to the neonatal units of two rural district hospitals in Rwanda after the 2012 launch of a national neonatal protocol and standards.

Methods: We retrospectively reviewed routinely collected data for all neonates ( 0 to 28 days) admitted to the neonatal units at Rwinkwavu and Kirehe District Hospitals from January 1, 2013 to December 31, 2014. Data on demographic and clinical characteristics, clinical management, and outcomes were analyzed using median and interquartile ranges for continuous data and frequencies and proportions for categorical data. Clinical management and outcome variables were stratified by birth weight and differences between low birth weight (LBW) and normal birth weight (NBW) neonates were assessed using Fisher's exact or Wilcoxon rank-sum tests at the $a=0.05$ significance level.
\end{abstract}

Results: A total of 1723 neonates were hospitalized over the two-year study period; $88.7 \%$ were admitted within the first $48 \mathrm{~h}$ of life, $58.4 \%$ were male, $53.8 \%$ had normal birth weight and $36.4 \%$ were born premature. Prematurity (27.8\%), neonatal infection (23.6\%) and asphyxia (20.2\%) were the top three primary diagnoses. Per national protocol, vital signs were assessed every $3 \mathrm{~h}$ within the first $48 \mathrm{~h}$ for $82.6 \%$ of neonates $(n=965 / 1168)$ and $93.4 \%$ $(n=312 / 334)$ of neonates with infection received antibiotics. The overall mortality rate was $13.3 \%(n=185 / 1386)$ and preterm/LBW infants had similar mortality rate to NBW infants (14.7 and $12.2 \%$ respectively, $p=0.131$ ). The average length of stay in the neonatal unit was 5 days.

Conclusions: Our results suggest that it is possible to provide specialized neonatal care for both LBW and NBW high-risk neonates in resource-limited settings. Despite implementation challenges, with the introduction of the neonatal care package and defined clinical standards these most vulnerable patients showed survival rates comparable to or higher than neighboring countries.

Keywords: Neonatal mortality, Neonate, Low birth weight, Prematurity, Quality of care, Mortality rate

\footnotetext{
* Correspondence: nymerab@gmail.com

${ }^{1}$ Partners In Health/Inshuti Mu Buzima (PIH/IMB), P.O. Box 3432, Kigali,

Rwanda

Full list of author information is available at the end of the article
}

(c) The Author(s). 2018 Open Access This article is distributed under the terms of the Creative Commons Attribution 4.0 International License (http://creativecommons.org/licenses/by/4.0/), which permits unrestricted use, distribution, and reproduction in any medium, provided you give appropriate credit to the original author(s) and the source, provide a link to the Creative Commons license, and indicate if changes were made. The Creative Commons Public Domain Dedication waiver (http://creativecommons.org/publicdomain/zero/1.0/) applies to the data made available in this article, unless otherwise stated. 


\section{Background}

Despite efforts to reduce neonatal mortality globally, in 2015 , nearly half of the 5.9 million under-five deaths occurred in the first 28 days of life [1] and eight of the 10 countries with the highest neonatal mortality rates are in sub-Saharan Africa (SSA) [2]. The main clinical causes of death include prematurity, infection, inadequate management of complications of pregnancy and delivery, and lack of quality care immediately after birth [1-4]. The majority of these deaths are preventable through evidence-based clinical interventions, such as antibiotic administration [5], oxygen therapy, continuous positive airway pressure [6] and caffeine treatment $[7,8]$. However, implementing these interventions in resource-limited settings can be challenging due to health system constraints, including limited equipment, lack of standardized protocols to guide neonatal management, insufficient training and support for clinical staff, and the shortage of pediatricians and neonatologists $[9,10]$.

In Rwanda, there has been a tremendous reduction in under-five mortality, which fell from 196 per 1000 live births in 2000 to 50 per 1000 live births in 2015, making the country one of the few in SSA to achieve the Millennium Development Goals for child mortality [11-14]. In addition, the neonatal mortality rate significantly declined from 41 per 1000 live births in 1990 to 17 per 1000 live births in 2016 [2]. Although national surveillance systems are able to provide population level data on neonatal survival [15], little data exists on the treatment and quality of care provided to high-risk newborns at health facilities, as well as predictors of clinical success in Rwanda or other countries in SSA. One study conducted in a rural Rwandan district hospital reported that over $60 \%$ of neonatal deaths occurred at presentation or shortly after admission and could be attributed in part to the lack of trained staff and lack of standard care practice supported by protocols [16]. Subsequently, the researchers identified the need for training staff and establishing protocols as vital for improving neonatal care.

The Rwanda Ministry of Health (MOH) developed a national neonatal care protocol and standards in partnership with a number of organizations, including Partners In Health/Inshuti Mu Buzima (PIH/IMB) - an international non-profit organization committed to improving health services in impoverished communities - and specialists from Boston Children's Hospital in Boston, USA. The neonatal care package was nationally adopted in 2012 with the goal of providing quality care to sick and preterm/low birth weight infants in rural district hospitals which lack specialist physicians $[17,18]$. The protocol was initially implemented and tested in the neonatal units of two rural PIH/IMB supported MOH district hospitals in 20102011. Details on the development and implementation of the newborn medicine program have been reported previously in Hansen et al's 2015 study [18]. The protocol implementation included roll-out of standardized medical records, quality indicators, and corresponding training materials. Here, we describe the presentation, clinical management, and outcomes of neonates after approximately 2 years of implementation of the neonatal care package. Neonates admitted between January 2013 and December 2014 to the neonatal units of the two rural district hospitals where the care package was first introduced were included. We also compared outcomes between low birth weight (LBW) and normal birth weight (NBW) neonates to detect any differences that may exist in the delivery of care to these patients. We aimed to highlight successes and gaps in the implementation of the national neonatal care package that could support quality care provision for neonates in Rwanda and similar settings.

\section{Methods}

\section{Study design and setting}

This retrospective cross-sectional study included neonates admitted to the neonatal units of PIH/IMB supported $\mathrm{MOH}$ Rwinkwavu and Kirehe District Hospitals (RDH, KDH). Both hospitals are located in rural areas of the Eastern Province of Rwanda and serve a total population of approximately 550,000 [19]. The initial roll-out involved international neonatal physician and nurse specialist support. After introduction, routine technical support has included support for training and ongoing mentorship of general practitioners and nurses working in the neonatal units by PIH/IMB-employed Rwandan nurse mentors. Visiting specialist physicians supporting $\mathrm{PIH} / \mathrm{IMB}$ 's medical education mission provided intermittent support. Additionally, PIH/IMB provided targeted support for essential equipment and consumables as part of health system strengthening.

At either of these two district hospitals, about 2700 to 2800 deliveries per year are recorded and roughly over $90 \%$ are referrals from health centers in the district hospitals' catchment areas. The average facility delivery rate in the Eastern Province is $88.8 \%$ [15]. When neonates are born in clinical distress or exhibit risk factors such as LBW, prematurity, sepsis or birth asphyxia, they are transferred to a neonatal unit for ongoing clinical management.

\section{Neonatal unit structure and function}

At the time of this study, seven to ten certified nurses or midwives staffed each neonatal unit. During the day shift, two nurses/midwives worked in the neonatology unit and one general practitioner (GP) supervised both the pediatric and neonatal units. Overnight, one to two GPs staffed the hospital and nurses/midwives in the neonatal units called them if in need of assistance. On average, there were 10 to 15 neonates in the neonatal unit at each hospital per day. 
Training and on-site mentorship were provided intermittently throughout the study period by an expert physician and nurse trainers to the physicians and nurses staffing the units. Available equipment in the neonatal units included beds for kangaroo mother/skin-to-skin care, syringe pumps, incubators, radiant warmers and phototherapy units since 2011 and bubble continuous positive airway pressure (bCPAP) machines had recently been introduced in January 2013.

\section{Study population, sources of data and analysis}

All neonates aged 0 to 28 days and admitted to the hospitals between January 1, 2013, and December 31, 2014 were included in our analysis. Data was extracted from patients' medical files using a standardized neonatal data collection form and entered into an electronic database by hired and trained non-clinical data officers supporting hospital monitoring and evaluation. Before data collection the officers completed a two-day orientation and training that focused on specific clinical terms used in the neonatal units and how to read patients' medical files and abstract data from the file to the data collection form. The data were extracted over a one-month period of time.

We analyzed a subset of data that contained neonatal demographic and clinical characteristics, clinical management and outcome information. The clinical management variables are based on the quality indicators, which were originally drafted for the protocol according to expert opinion and literature review and later modified to address topics identified as challenging by hospital clinical staff. The indicators aim to monitor key aspects of neonatal care provision per protocol, with a focus on the needs of sick, LBW and preterm neonates $[17,18]$. The indicators tracked the monitoring of vital signs, thermoregulation, hypoglycemia, administration of antibiotics for infectious diseases, fluid electrolytes and nutrition, and respiratory distress and included targets for high quality (Table 1). Cut-off point for hypoglycemia changed during the study period. Originally, low blood sugar was defined as less than $40 \mathrm{mg} / \mathrm{dl}$. The definition was eventually changed to be less than $45 \mathrm{mg} / \mathrm{dl}$ in order to support more cautious clinical management. It took time for the new definition to be applied, so for the purposes of our analysis we used the range of less than 40 or less than $45 \mathrm{mg} / \mathrm{dl}$ to define low blood sugar.

For this study, the antibiotic indicator is limited to antibiotics provided in the first $24 \mathrm{~h}$ of therapy. For routine monitoring and evaluation, it was most feasible to assess all charts with antibiotic provision, rather than algorithmic exclusions of those who were ruled out within $72 \mathrm{~h}$. Additionally, medication safety is a critical issue, particularly when introducing treatments in a neonatal population which can require dilution calculations. Therefore, the routinely monitored indicator was adapted to the version included here. Correct dose and interval for the first $24 \mathrm{~h}$ of therapy was determined according to the national neonatal protocol [17]. The actual dose administered was compared to the calculated correct dose using the

Table 1 Quality indicators definitions and targets

\begin{tabular}{|c|c|c|}
\hline Category & Indicator Definitions & Targets \\
\hline Vital signs & $\begin{array}{l}\text { Percent of patient records in which vital signs are documented } \\
\text { on average every } 3 \mathrm{~h} \text { within the first } 48 \mathrm{~h} \text { of admission }\end{array}$ & $\begin{array}{l}15 \text { (every } 3 h \times 48 h \text { with possibility of one less on } \\
\text { day of admission) per patient and } 80 \% \text { overall. }\end{array}$ \\
\hline \multirow[t]{2}{*}{ Thermoregulation } & $\begin{array}{l}\text { Percent of neonates who have first temperature documented } \\
\text { within } 30 \text { min of admission to neonatal unit }\end{array}$ & $80 \%$ \\
\hline & $\begin{array}{l}\text { Percent of neonates with documented first temperature after } \\
\text { admission }<36.0^{\circ} \mathrm{C} \text { having temperature improve to }>36.0^{\circ} \mathrm{C} \text { in } \leq 2 \mathrm{~h}\end{array}$ & $80 \%$ \\
\hline Hypoglycemia ${ }^{a}$ & $\begin{array}{l}\text { Percent of neonates with documented blood sugar }<40-<45 \mathrm{mg} / \mathrm{dL} \\
\text { who had blood sugar level improve to }>40->45 \mathrm{mg} / \mathrm{dL} \text { within } 1 \mathrm{~h}\end{array}$ & $80 \%$ \\
\hline Infectious disease & $\begin{array}{l}\text { Percent of neonates who received antibiotics (ampicillin and gentamicin) } \\
\text { at correct dose and interval for first } 24 \mathrm{~h} \text { of therapy }\end{array}$ & $80 \%$ \\
\hline $\begin{array}{l}\text { Fluid electrolytes } \\
\text { and nutrition }\end{array}$ & $\begin{array}{l}\text { Percent of neonates admitted to neonatal unit within first } 48 \text { h of life and } \\
\text { remain in unit until at least } 2 \text { weeks of age who regain their birth weight } \\
\text { by }<2 \text { weeks of age }\end{array}$ & $80 \%$ \\
\hline \multirow[t]{2}{*}{ Respiratory } & $\begin{array}{l}\text { Percent of neonates with } \mathrm{BW}<1.5 \mathrm{~kg} \text { or } \mathrm{GA}<33 \text { weeks for whom } \\
\text { methylxanthine treatment (caffeine or aminophylline) is prescribed }\end{array}$ & $80 \%$ \\
\hline & $\begin{array}{l}\text { Percent of preterm/LBW neonates eligible for CPAP who are started on CPAP } \\
\text { within } 2 \mathrm{~h} \text { of life (eligibility criteria: } \mathrm{BW}<2 \mathrm{~kg} \text { or } \mathrm{GA}<33 \text { weeks and any degree } \\
\text { of respiratory distress }-\mathrm{O}_{2} \text { saturation } \leq 90 \% \text { oxygen requirement and/or RR } \geq 50 \\
\text { and/or grunting/flaring/retractions) }\end{array}$ & $90 \%$ \\
\hline
\end{tabular}

${ }^{\circ} \mathrm{C}$ degrees centigrade, $\mathrm{mg} / \mathrm{dL}$ milligrams per deciliter, $B W$ birth weight, $L B W$ low birth weight, $\mathrm{kg}$ kilogram, GA gestational age, $O 2$ Oxygen, $R R$ respiratory rate, CPAP continuous positive airway pressure

${ }^{a}$ Cut-off point for hypoglycemia changed during the study period. Originally, low blood sugar was defined as less than 40 mg/dl. The definition was eventually changed to be less than $45 \mathrm{mg} / \mathrm{dl}$. It took time for the new definition to be applied, so for the purposes of our analysis we used the range of less than 40 or less than $45 \mathrm{mg} / \mathrm{dl}$ to define low blood sugar 
neonate's birth weight, and administration interval was calculated using the time of medication administration as documented. In the two participating hospitals, the quality indicators were reviewed quarterly to monitor progress and inform quality improvement initiatives. All extracted data was verified for accuracy and completeness during routine audits performed by the MOH's and PIH/IMB's monitoring and evaluation teams.

We report demographic and clinical characteristics, clinical management and outcome variables using frequencies and proportions for categorical data and medians with interquartile ranges (IQR) for continuous data. We stratified the data by birth weight and used Fisher's exact tests for categorical variables and Wilcoxon rank-sum tests for continuous variables to compare clinical management and outcomes variables between LBW and NBW neonates at the $\alpha=0.05$ significance level. NBW included neonates with a birth weight of $\geq 2500$ g. LBW included neonates with a birth weight $<2500 \mathrm{~g}$, with the sub-categories of extremely LBW neonates $(<1000 \mathrm{~g})$, very LBW neonates (1000 to $1499 \mathrm{~g}$ ) and LBW neonates (1500 to $2499 \mathrm{~g}$ ). Although not used for stratification, gestational age is reported for some variables and was categorized as term ( $\geq 37$ weeks) and preterm ( $<37$ weeks). Outcomes included the number of neonates discharged, transferred, absconded (defined as leaving against medical advice) and deceased at the end of the study period, as well as weight at discharge and length of stay in the neonatal unit. Missing data were analyzed using a pairwise deletion for the missing data at random. Data were analyzed using Stata v13 (College Station, TX: StataCorp LP).

\section{Results}

A total of 1723 neonates were admitted to the two district hospital neonatology units; $49.7 \%(n=856)$ to Kirehe and 50.3\% ( $n=867)$ to Rwinkwavu (Table 2), respectively. Admission age was recorded for 1684 neonates; $88.7 \%(n=1493)$ were admitted within the first $48 \mathrm{~h}$ of life and $58.4 \%(n=949$ of 1624$)$ were males. Birth weight was recorded for 1518 neonates and gestational age recorded for 1528 ; $46.2 \%(n=501)$ were LBW and $53.8 \%(n=817)$ were NBW, and $36.4 \%(n=556)$ were preterm and $63.6 \%(n=972)$ were term. The top three primary diagnoses (among the 1663 neonates with recorded diagnoses) were prematurity $(27.8 \%, n=463)$, neonatal infection $(23.6 \%, n=392)$, and asphyxia $(20.2 \%, n=336)$.

For clinical management during a hospital stay, $82.6 \%$ ( $n=965$ of 1168) of neonates had their vital signs checked and documented at least 15 times within the first $48 \mathrm{~h}$ of their hospital admission (Table 3). For thermoregulation, $55.0 \%$ ( $n=812$ of 1476) of neonates had their initial temperature measured within $30 \mathrm{~min}$ of admission; $29.4 \% \quad(n=435$ of 1480$)$ had an initial
Table 2 Socio-demographic and clinical characteristics of neonates admitted to neonatology units at two rural district hospitals in Rwanda ( $N=1723)$

\begin{tabular}{|c|c|c|}
\hline & $\mathrm{n}$ & $\%$ \\
\hline \multicolumn{3}{|l|}{ Hospital } \\
\hline Kirehe & 856 & 49.7 \\
\hline Rwinkwavu & 867 & 50.3 \\
\hline Age at admission (days) & $N=1621$ & \\
\hline$<1$ & 1140 & 70.3 \\
\hline $1-3$ & 310 & 19.1 \\
\hline $4-7$ & 54 & 3.3 \\
\hline $8-28$ & 117 & 7.2 \\
\hline Admitted in first $48 \mathrm{~h}$ of life & $N=1684$ & \\
\hline Yes & 1493 & 88.7 \\
\hline No & 191 & 11.3 \\
\hline Gender & $N=1624$ & \\
\hline Male & 949 & 58.4 \\
\hline Female & 675 & 41.6 \\
\hline Birth weight (grams) & $N=1518$ & \\
\hline Low birth weight $(<2500)$ & 701 & 46.2 \\
\hline LBW ( $\geq 1500-<2500)$ & 528 & 34.8 \\
\hline Very LBW $(\geq 1000-<1500)$ & 139 & 9.2 \\
\hline Extremely LBW $(<1000)$ & 34 & 2.2 \\
\hline Normal birth weight $(\geq 2500)$ & 817 & 53.8 \\
\hline Gestational age (weeks) & $N=1528$ & \\
\hline Preterm $(<37)$ & 556 & 36.4 \\
\hline Preterm $(\geq 33$ to $<37$ ) & 391 & 25.6 \\
\hline Very preterm $(<33)$ & 165 & 10.8 \\
\hline Term $(\geq 37)$ & 972 & 63.6 \\
\hline Primary diagnosis & $N=1663$ & \\
\hline Prematurity & 463 & 27.8 \\
\hline Neonatal infection & 392 & 23.6 \\
\hline Asphyxia at birth/low APGAR score/HIE & 336 & 20.2 \\
\hline Respiratory distress/apnea & 113 & 6.8 \\
\hline Low birth weight & 95 & 5.7 \\
\hline Poor feeding & 51 & 3.1 \\
\hline Malformation & 36 & 2.2 \\
\hline Convulsion & 28 & 1.7 \\
\hline Pneumonia & 21 & 1.3 \\
\hline Jaundice & 13 & 0.8 \\
\hline Hypothermia & 10 & 0.6 \\
\hline Hypoglycemia & 8 & 0.5 \\
\hline Others & 97 & 5.8 \\
\hline
\end{tabular}

LBW Low birth weight, APGAR A measurement of Appearance, Pulsation, Grimace, Activity and Respiration, HIE Hypoxic Ischemic Encephalopathy 
Table 3 Clinical management and interim outcomes of neonates with birth weight recorded upon admission to neonatology units at two rural district hospitals in Rwanda $(N=1518)$

\begin{tabular}{|c|c|c|c|c|c|c|c|}
\hline & \multicolumn{2}{|c|}{$\begin{array}{l}\text { All neonates } \\
(N=1518)\end{array}$} & \multicolumn{2}{|c|}{$\begin{array}{l}\text { Low birth weight } \\
(<2500 \mathrm{~g}) \\
(N=701)\end{array}$} & \multicolumn{2}{|c|}{$\begin{array}{l}\text { Normal birth weight } \\
(\geq 2500 \mathrm{~g}) \\
(N=817)\end{array}$} & \multirow[t]{2}{*}{$p$-value } \\
\hline & $n$ & $\%$ & $n$ & $\%$ & $n$ & $\%$ & \\
\hline \multicolumn{8}{|l|}{ Vital signs } \\
\hline Vital signs checked every $3 \mathrm{~h}$ for the first $48 \mathrm{~h}$ of admission & \multicolumn{2}{|c|}{$N=1168$} & \multicolumn{2}{|c|}{$N=556$} & \multicolumn{2}{|c|}{$N=612$} & \\
\hline Yes ( $\geq 15$ times) & 965 & 82.6 & 478 & 86.0 & 487 & 79.6 & 0.004 \\
\hline No (< 15 times) & 203 & 17.4 & 78 & 14.0 & 125 & 20.4 & \\
\hline \multicolumn{8}{|l|}{ Thermoregulation } \\
\hline Initial temperature measured within 30 min of admission & \multicolumn{2}{|c|}{$N=1476$} & \multicolumn{2}{|c|}{$N=683$} & \multicolumn{2}{|c|}{$N=793$} & \\
\hline Yes & 812 & 55.0 & 367 & 53.7 & 445 & 56.1 & 0.373 \\
\hline No & 664 & 45.0 & 316 & 46.3 & 348 & 43.9 & \\
\hline Initial temperature $<36^{\circ} \mathrm{C}$ & \multicolumn{2}{|c|}{$N=1480$} & \multicolumn{2}{|c|}{$N=684$} & \multicolumn{2}{|c|}{$N=796$} & \\
\hline Yes & 435 & 29.4 & 249 & 36.4 & 186 & 23.4 & $<0.001$ \\
\hline No & 1045 & 70.6 & 435 & 63.6 & 610 & 76.6 & \\
\hline $\begin{array}{l}\text { If initial temperature }<36{ }^{\circ} \mathrm{C} \text {, temperature improved from }<36{ }^{\circ} \mathrm{C} \text { to }>36{ }^{\circ} \mathrm{C} \\
\text { within } 2 \mathrm{~h}\end{array}$ & \multicolumn{2}{|c|}{$N=402$} & \multicolumn{2}{|c|}{$N=234$} & \multicolumn{2}{|c|}{$N=168$} & \\
\hline Yes & 156 & 38.8 & 99 & 42.3 & 57 & 33.9 & 0.097 \\
\hline No & 246 & 61.2 & 135 & 57.7 & 111 & 66.1 & \\
\hline \multicolumn{8}{|l|}{ Hypoglycemia } \\
\hline Low blood sugar $(<40 /<45 \mathrm{mg} / \mathrm{dl})$ & \multicolumn{2}{|c|}{$N=871$} & \multicolumn{2}{|c|}{$N=418$} & \multicolumn{2}{|c|}{$N=453$} & \\
\hline Yes & 31 & 3.6 & 21 & 5.0 & 10 & 2.2 & 0.028 \\
\hline No & 840 & 96.4 & 397 & 95.0 & 443 & 97.8 & \\
\hline If blood sugar low, improved to $>40 />45 \mathrm{mg} / \mathrm{dl}$ within $1 \mathrm{~h}$ & $N=12$ & & $N=7$ & & $N=5$ & & \\
\hline Yes & 2 & 16.7 & 1 & 14.3 & 1 & 20 & $>0.999$ \\
\hline No & 10 & 83.3 & 6 & 85.7 & 4 & 80 & \\
\hline \multicolumn{8}{|l|}{ Infectious diseases } \\
\hline Received antibiotics (ampicillin and gentamicin) ${ }^{a}$ & \multicolumn{2}{|c|}{$N=334$} & \multicolumn{2}{|c|}{$N=69$} & $N=26$ & & \\
\hline Yes & 312 & 93.4 & 68 & 98.6 & 244 & 92.1 & 0.053 \\
\hline No & 22 & 6.6 & 1 & 1.4 & 21 & 7.9 & \\
\hline Ampicillin received at correct dose and interval & $N=30$ & & $N=6$ & & $N=23$ & & \\
\hline Yes & 204 & 67.8 & 36 & 54.6 & 168 & 71.5 & 0.009 \\
\hline No & 97 & 32.2 & 30 & 45.4 & 67 & 28.5 & \\
\hline Gentamicin received at correct does and interval & $N=28$ & & $N=6$ & & $N=22$ & & \\
\hline Yes & 146 & 51.0 & 23 & 35.9 & 123 & 55.4 & 0.006 \\
\hline No & 140 & 49.0 & 41 & 64.1 & 99 & 44.6 & \\
\hline Fluid electrolytes and nutrition & & & & & & & \\
\hline Regained birth weight within 2 weeks ${ }^{b}$ & $N=22$ & & $N=$ & & $N=56$ & & \\
\hline Yes & 126 & 56.0 & 88 & 52.1 & 38 & 67.9 & 0.044 \\
\hline No & 99 & 44.0 & 81 & 47.9 & 18 & 32.1 & \\
\hline Respiratory distress & & & & & & & \\
\hline Received caffeine $^{c}$ & & & $N=5$ & & & & \\
\hline Yes & & & 206 & 37.6 & & & \\
\hline No & & & 342 & 62.4 & & & \\
\hline
\end{tabular}


Table 3 Clinical management and interim outcomes of neonates with birth weight recorded upon admission to neonatology units at two rural district hospitals in Rwanda ( $N=1518)$ (Continued)

\begin{tabular}{|c|c|c|c|c|c|c|c|}
\hline & \multicolumn{2}{|c|}{$\begin{array}{l}\text { All neonates } \\
(N=1518)\end{array}$} & \multicolumn{2}{|c|}{$\begin{array}{l}\text { Low birth weight } \\
(<2500 \mathrm{~g}) \\
(N=701)\end{array}$} & \multicolumn{2}{|c|}{$\begin{array}{l}\text { Normal birth weight } \\
(\geq 2500 \mathrm{~g}) \\
(N=817)\end{array}$} & \multirow[t]{2}{*}{$p$-value } \\
\hline & $n$ & $\%$ & $n$ & $\%$ & $n$ & $\%$ & \\
\hline Received oxygen therapy & \multicolumn{2}{|c|}{$N=1491$} & \multicolumn{2}{|l|}{$N=690$} & \multicolumn{2}{|l|}{$N=801$} & \\
\hline Yes & 603 & 40.4 & 278 & 40.3 & 325 & 40.6 & 0.916 \\
\hline No & 888 & 59.6 & 412 & 59.7 & 476 & 59.4 & \\
\hline Method of oxygen therapy & \multicolumn{2}{|l|}{$N=540$} & \multicolumn{2}{|l|}{$N=247$} & \multicolumn{2}{|l|}{$N=293$} & \\
\hline Mask/nasal cannula & 449 & 83.1 & 185 & 74.9 & 264 & 90.1 & $<0.001$ \\
\hline bCPAP & 91 & 16.9 & 62 & 25.1 & 29 & 9.9 & \\
\hline If eligible, received bCPAPd & & & \multicolumn{2}{|l|}{$N=107$} & & & \\
\hline Yes & & & 13 & 12.2 & & & \\
\hline No & & & 94 & 87.8 & & & \\
\hline Duration of oxygen therapy in hours & Median & IQR & Median & IQR & Median & IQR & \\
\hline Mask/nasal cannula & 24 & 20,96 & 48 & 24,120 & 24 & 14,72 & 0.050 \\
\hline bCPAP & 60 & 24,120 & 72 & 24,144 & 48 & 24,72 & 0.093 \\
\hline
\end{tabular}

${ }^{\mathrm{a}}$ For neonates with primary diagnosis of infection; ${ }^{\mathrm{b}}$ Restricted to neonates that were hospitalized for at least 2 weeks; ${ }^{\mathrm{c}}$ Restricted to preterm neonates $\left(<33\right.$ weeks) or LBW $(<1500 \mathrm{~g}) ;{ }^{\mathrm{d}}$ Restricted to preterm $(<33$ weeks) or LBW $(<1500 \mathrm{~g})$ neonates with any sign of respiratory distress

${ }^{\circ} \mathrm{C}$ degrees centigrade, $\mathrm{gm} / \mathrm{dl}$ grams per decilitre, $b C P A P$ bubble continuous positive airway pressure, IQR inter-quartile range

temperature below $36{ }^{\circ} \mathrm{C}$ recorded, and $38.8 \%(n=156$ of 402) had an initial temperature below $36^{\circ} \mathrm{C}$ improve to be greater than $36^{\circ} \mathrm{C}$ within $2 \mathrm{~h}$ of the time the initial temperature was taken. Hypoglycemia was also assessed; $3.6 \%$ (31 of 871 ) of neonates had low blood sugar levels (either $<40$ or $<45 \mathrm{mg} / \mathrm{dl}$ ) and $16.7 \%$ ( $n=2$ of 12$)$ neonates with low blood sugar improved to have a blood sugar measurement of either $>40$ or $>45 \mathrm{mg} / \mathrm{dl} \mathrm{docu-}$ mented within the hour.

Of the 334 neonates with a primary diagnosis of infection, $93.4 \%(n=312)$ received antibiotics. Among the 301 neonates who had data recorded on ampicillin administration and the 286 neonates who had data recorded on gentamicin administration, $67.8 \%(n=204)$ and $51.0 \%(n=146)$ received the correct dose at the correct time interval, respectively. Of 225 neonates who stayed in the hospital for at least 14 days, $56.0 \%(n=126)$ regained their birth weight within the 2 weeks. With regard to respiratory support, $40.4 \%(n=603)$ of 1491 neonates received oxygen therapy. Of 540 neonates with oxygen therapy method documented, $83.1 \%(n=449)$ received mask or nasal cannula and $16.9 \%(n=91)$ received bCPAP. Of the 107 preterm/low birth weight neonates meeting eligibility criteria for bCPAP (born $<1500 \mathrm{~g}$ or $<$ 33 weeks and showing any signs of respiratory distress), $12.2 \%(n=13)$ received bCPAP. Among the 548 neonates born $<1500 \mathrm{~g}$ or $<33$ weeks (meeting eligibility criteria), $37.6 \%(n=206)$ received caffeine citrate.

When results were stratified by birth weight, there was no evidence of differences in clinical management between LBW and NBW neonates for the following variables: initial temperature measured within $30 \mathrm{~min}$ of admission $(p=0.373)$, improvement in initial temperature from $<36{ }^{\circ} \mathrm{C}$ to $>36{ }^{\circ} \mathrm{C}(p=0.097)$, improvement in low blood sugar levels to normal levels $(p>0.999)$, and administration of oxygen therapy ( $p=0.916)$ (Table 3$)$.

However, we observed significant differences in the clinical management of LBW and NBW neonates for a number of variables. Vital signs were checked at least 15 times within the first $48 \mathrm{~h}$ of admission for $86.0 \%(n=$ 478 of 556$)$ of LBW neonates and $79.6 \%(n=487$ of 612$)$ of NBW neonates $(p=0.004)$. Antibiotics were administered to $98.6 \%$ ( $n=68$ or 69 ) of LBW neonates with infection and $92.1 \%(n=244$ of 265$)$ of NBW neonates with infection $(p=0.053)$. Correct dosage and interval of ampicillin was provided to $54.6 \%(n=36$ of 66$)$ LBW neonates and $71.5 \%(n=168$ of 235$)$ NBW neonates $(p=$ $0.009)$, and to $35.9 \%(n=23$ of 64$)$ LBW neonates and $55.4 \%$ ( $n=123$ of 222 ) NBW neonates for gentamicin $(p$ $=0.006)$. Approximately half $(52.1 \%, n=88$ of 169$)$ of LBW neonates regained birth weight within 2 weeks, compared to $67.9 \%(n=38$ of 56$)$ of NBW neonates $(p=$ 0.044). For method of oxygen therapy, LBW neonates were more likely to receive bCPAP compared to NBW neonates $(25.1 \%, n=62$ of 247 and $9.9 \%, n=29$ of 293 , respectively, $p<0.001)$. LBW neonates received oxygen for a longer duration, whether on mask/nasal cannula (median $=48$ h, IQR: $24-120, p=0.050$ ) or bCPAP (median $=72$ h, IQR: $24-144, p=0.093$ ) compared to NBW neonates, who used mask/nasal cannula for a median of $24 \mathrm{~h}$ (IQR 14-72) and bCPAP for a median of $48 \mathrm{~h}$ (IQR 24-72, $p=0.093)$. 
Overall, $83.3 \%(n=1162)$ of the neonates were discharged, $13.3 \%(n=185)$ died, $2.3 \%(n=32)$ transferred and $0.5 \%(n=7)$ absconded (Table 4$)$. The top three primary diagnoses among the 183 deceased neonates with primary diagnosis recorded were asphyxia (36.1\%, $n=66)$, prematurity $(29.5 \%, n=54)$, and respiratory distress $(13.1 \%, n=24)$. There was no evidence of differences in outcomes between LBW and NBW neonates $(p=0.131)$. The overall median length of stay in the neonatal unit was 5 days (IQR: $2-10)$. The length of stay was significantly longer for LBW neonates (median = 7 days, IQR: 2-14) compared to NBW neonates (median $=4$ days, IQR: $2-7, p<0.001)$. This remained true when stratified by those who survived to discharge (median = 8 days, IQR: $3-18$ vs. median $=5$, IQR: $2-8, p<0.001$ ) and those who did not (median $=2$ days, IQR: $0-2$ vs median $=1$ day, IQR: $0-2, p=0.003$ ). Of the neonates who were discharged, 477 were low birthweight at admission, and 94.1\% $(n=449)$ remained under $2500 \mathrm{~g}$ when they were discharged, while of the 536 neonates of normal birthweight at admission, $12.3 \%(n=66)$ were under $2500 \mathrm{~g}$ at discharge $(p<0.001)$.

\section{Discussion}

Neonatal care provision in rural resource-limited settings is a challenge for many countries in SSA and in the early stages of introduction [18-25]. Hansen et al. (2015) showed that while neonatal care may be considered a specialized clinical service, it can be standardized and implemented in rural district hospitals in Rwanda [18]. Here, we show clinical management and quality of neonatal care in two rural district hospitals that were guided by this neonatal care package. Our study had a number of key results.

First, we found that the demographic and clinical characteristics of our neonatal sample were similar to those reported in other studies. Consistent with studies showing higher and earlier hospital admission rates for neonates within the first 7 days of birth and higher morbidity rates among male neonates [2, 20, 26, 27], most neonates in our study were admitted to the neonatal units within the first $48 \mathrm{~h}$ after birth and were males. Prematurity, infection and asphyxia were the top three causes of infant illnesses, mirroring findings reported in other parts of SSA [1, 20, 27, 28]. These patterns show that more research into the underlying causes of neonatal illness in resource-limited settings is needed, as are interventions that can address these persistent challenges.

Second, our assessment of clinical management and outcome variables shows that it is possible to provide care to high-risk neonates in this setting with reasonable patient outcomes, particularly when compared to those of other countries in the region [27]. A study on the use of medicines in 104 developing and transitional countries from 1990 to 2009 found that the percentage of patients receiving antibiotics increased from 45 to $54 \%$ over this 20-year period [29] and the management of prematurity-related complications using antibiotics was only $50 \%$ [27]. Additionally, the majority of LBW neonates, who are at the highest risk of morbidity, were more likely to receive care according to protocol compared to

Table 4 Outcomes of neonates with birth weight recorded upon admission to neonatology units at two rural district hospitals in Rwanda $(N=1518)$

\begin{tabular}{|c|c|c|c|c|c|c|c|}
\hline & \multicolumn{2}{|c|}{ All neonates $(N=1518)$} & \multicolumn{2}{|c|}{ Low birth weight $(<2500 \mathrm{~g})(\mathrm{N}=701)$} & \multicolumn{2}{|c|}{ Normal birth weight $(\geq 2500 \mathrm{~g})(N=817)$} & \multirow[t]{2}{*}{$p$-value } \\
\hline & $\mathrm{n}$ & $\%$ & $\mathrm{n}$ & $\%$ & $\mathrm{n}$ & $\%$ & \\
\hline Outcome & $N=1386$ & & $N=626$ & & $N=760$ & & \\
\hline Discharged & 1162 & 83.3 & 522 & 83.4 & 640 & 84.2 & 0.131 \\
\hline Transferred & 32 & 2.3 & 11 & 1.8 & 21 & 2.8 & \\
\hline Absconded & 7 & 0.5 & 1 & 0.2 & 6 & 0.8 & \\
\hline Died & 185 & 13.3 & 92 & 14.7 & 93 & 12.2 & \\
\hline Length of stay ${ }^{a}$ & Median & IQR & Median & IQR & Median & IQR & \\
\hline All neonates & 5 & 2,10 & 7 & 2,14 & 4 & 2,7 & $<0.001$ \\
\hline Discharged & 6 & 2,11 & 8 & 3,18 & 5 & 2,8 & $<0.001$ \\
\hline Transferred & 3 & $0.5,9.5$ & 3.5 & 0,11 & 2.5 & $0.5,7.5$ & 0.722 \\
\hline Absconded & 7 & 1,15 & 21 & 21,21 & 5 & 1,8 & 0.134 \\
\hline Died & 1 & 0,3 & 2 & 1,4 & 1 & 0,2 & 0.003 \\
\hline Weight at discharge (grams) ${ }^{b}$ & $N=1013$ & & $N=477$ & & $N=536$ & & \\
\hline$<2500$ & 515 & 50.8 & 449 & 94.1 & 66 & 12.3 & $<0.001$ \\
\hline$\geq 2500$ & 498 & 49.2 & 28 & 5.9 & 470 & 87.7 & \\
\hline
\end{tabular}

${ }^{\mathrm{a}}$ Only reported for neonates that had an admission date and outcome date or age at admission and age at discharge; ${ }^{\mathrm{b}}$ Limited to neonates who were discharged 
NBW neonates in a number of clinical domains, including close monitoring (vital sign measurement) and duration on oxygen therapy, which are important interventions to reduce clinical deterioration in this population $[5,30]$. LBW neonates also stand to gain the greatest impact from bCPAP for the treatment of respiratory distress syndrome $[5,21]$ and their higher rates of bCPAP use and longer duration on oxygen therapy showed possible prioritization of their needs by health care workers, particularly in a staff-constrained environment. The transfer rate for neonates was low and impressively similar to a study conducted at a Rwandan provincial hospital (which is supposed to have a higher level of care than district hospitals) which reported a drop-in neonate transfer rates from 50 to $2 \%$ in 1 year after a series of quality improvement interventions were implemented, including introduction of standardized treatment procedures [16].

Finally, and most importantly, the overall neonatal unit survival rate for both LBW and NBW neonates was higher than results reported from similar settings and did not differ across birth weight categories [23, 26, 31]. This study is descriptive and limits any assessment of causality; however, given that no standardized care for sick and preterm infants was provided before the establishment of the neonatal care unit and these infants were previously being referred to the one national referral hospital in the country, it demonstrates that neonatal care can be provided in a decentralized manner with the appropriate investment in neonatal unit establishment, vastly improving population access to this essential service. Availability and ease of protocol use, presence of essential equipment and medications, along with intermittent training and mentorship for providers likely influenced the quality of care for high-risk neonates in these hospitals $[32,33]$. Asphyxia was a leading diagnosis among those who died, which could indicate a greater need to target improvement efforts during delivery and immediately after birth and underscore the need for interdepartmental coordination within the hospital across maternity, delivery, and neonatal units. Further research assessing the impact of the neonatal care package on population level neonatal mortality, as well as assessment of the optimal content and level of complexity for rural district hospitals is warranted.

Despite these successes, there is still a need to improve treatment practices to meet quality indicator targets and further improve neonatal outcomes. These targets were intentionally set high to serve as a goal for quality improvement efforts. Based on our results, management of vital signs measurement, weight gain, administration of antibiotics and thermoregulation measurement of temperature within the first $30 \mathrm{~min}$ of admission were close to the targets, while other indicators, such as improved temperature within $2 \mathrm{~h}$ of admission, caffeine administration, improved blood sugar levels and weight gain were not. These results can be used to inform the design and goals of quality improvement initiatives so they can be most effective. For example, while LBW infants had relatively strong outcomes, a low percentage of the bCPAP-eligible LBW/preterm neonates received bCPAP therapy, indicating further potential gains in neonatal outcomes that could be made. While this gap could be exaggerated due to the fact that bCPAP was introduced in first month of the study period and may have improved with increasing health care worker comfort and practice over time during the study period, a gap in bCPAP implementation was also noted by a second bCPAP-focused study from an overlapping time period [34]. Therefore, this area has been targeted for quality improvement since the completion of this study.

In addition, while antibiotics were administered to neonates diagnosed with infections, there is room for improvement in the timing and dosage of these antibiotics. As these data were used for quarterly data review with the clinical teams, quality improvement problem analyses found a number of challenges with hospital level neonatal antibiotic administration. The dose has to be selected based on gestational age/birth weight and a weight-based dose had to be correctly calculated. Some providers new to neonatal care, or not providing neonatal care routinely, found this selection challenging, and more NBW neonates received correct administration of gentamicin. Staff shortages and high patient volume could be challenges to administering antibiotics on-time. There is also room for improvement around documentation and recordkeeping. Further exploration into the issue should be pursued by asking the neonatal nurses about the documentation processes they follow and challenges they face. Observation of documentation practices can also be done if possible. Strategies for improving documentation should be designed and implemented as a collaborative effort between nurses and researchers to better support uptake and sustainability.

Our results also show that quality improvement efforts need to be directed at NBW neonates as well. We found that $12 \%$ of neonates admitted to the hospital with a normal birthweight were discharged weighing less than $2500 \mathrm{~g}$. While this could be focused among neonates born just above the LBW cut-off experiencing normal weight loss in the first few days of life with short-course hospitalizations, this may indicate that supporting neonatal growth and nutrition was challenging. Taking the full context into consideration, this may be due to a family's inability to pay for the costs of hospitalization or because parents are unable to leave other children at home or be absent from work to be in the hospital. Therefore, this finding warrants further investigation into how to support families comprehensively, and more interventions, such as social support 
packages that can address financial challenges, are needed to ensure these high-risk vulnerable neonates get the treatment they need to thrive.

Health systems challenges may have also influenced the ability to reach certain indicator targets. Not being able to apply to full protocol due to staffing shortages, high turnover of trained staff, misaligned rotations where providers not trained in neonatal care are assigned to the neonatal unit and stock outs of drug and laboratory reagents may have impacted the quality of care provided in the neonatal units $[4,28]$. We recommend implementation of quality improvement projects that target the national indicators, accurate and timely documentation of management and outcomes, revision of the rotation system to ensure the nurses staffing the neonatal unit have necessary training and skills for neonatal management and harmonizing internal transfers of neonates to ensure continuity of care as activities that can address the challenges we see in our neonatal units [35].

It is important to note that the care protocol was not introduced as an isolated document - when first introduced, it was paired with medical record introduction, quality indicators, and institutionalized training and mentorship. More broadly, will-building across hospital leadership and health care providers to establish a neonatal unit with designated physician and nursing staff and management was critical to providing a setting in which this introduction could have any measure of success. The district hospitals have both adopted district-based quality improvement initiatives in response to these data which were reviewed quarterly, including projects aimed at accurate and timely documentation of management and outcomes, revision of the rotation system to ensure the nurses staffing the neonatal unit have necessary training and skills for neonatal management, and coordination of internal transfer processes of neonates to ensure continuity of care [36].

This study has several limitations. First, as a retrospective cross-sectional study over a two-year time period, we were unable to assess variations in quality across time and potential relationships with intervening variables such as those related to constrained health systems and timing of introduction of new initiatives such as bCPAP listed previously. We do not have data for year-to-year variations in the indicators. Data variation over a longer period would have to take issues like staffing and policy changes into consideration, which were outside the scope of this study. Along the same vein, we chose not to examine trends in performance on the quality indicators because we chose to focus on the current level of performance. Trends in performance in relation to specific quality improvement interventions could be the subject of future studies. Additionally, some of the diagnoses, such as infection and asphyxia, require laboratory evaluations for definitive diagnosis that were not available at this district hospital level [22]. However, we believe the clinical management to be appropriate if the provider closely followed the guidance for clinical management of a given suspected diagnosis, as they are designed to leverage the diagnostic resources available in these low-resource settings. Finally, another limitation was missing data, which we believe was due to the high documentation burden placed on the nurses and physicians staffing the neonatal units. Approximately $12 \%$ of the records were missing data and not included in the analysis of clinical management and interim outcomes shown in Table 3. Results were reported only for neonates who had a birth weight recorded upon admission to the neonatology unit. While missing data limited the ability to which we were able to generalize our study findings, we are still able to present outcomes and discuss our experiences of treating small and sick neonates in accordance with a national neonatal care package. Periodic data quality checks and training with an emphasis on the importance of proper documentation in clinical charts could improve data quality. In addition, future plans to implement electronic medical record systems in the hospital units could also help address these data quality gaps.

\section{Conclusions}

This study demonstrates the feasibility of specialized neonatal care in resource-limited settings supported by a standardized national package of services including infrastructure, training and supplies, as well as the implementation of a national neonatal protocol. LBW neonates received higher quality care compared to NBW and overall mortality rates were lower or comparable to other urban and tertiary hospital settings among both categories. However, gaps in care management remain that should be addressed in order to achieve further gains in morbidity and mortality. We recommend quality improvement efforts to address lagging indicators as well as continuous training and mentorship to ensure new providers are empowered with the tools necessary to provide high quality care to these vulnerable newborns and families.

\footnotetext{
Abbreviations

bCPAP: Bubble continuous positive airway pressure; GP: General practitioner; IQR: Interquartile range; KDH: Kirehe District Hospital; LBW: Low birth weight; NBW: Normal birth weight; ${ }^{\circ} \mathrm{C}$ : Degrees centigrade; PIH/IMB: Partners In Health/ Inshuti Mu Buzima; RDH: Rwinkwavu District Hospital; $\mathrm{MOH}$ : Ministry of Health; SSA: Sub-Saharan Africa
}

\section{Acknowledgements}

This study was developed under the Partners In Health/Inshuti Mu Buzima Intermediate Operational Research Training Program, developed and facilitated by Bethany Hedt-Gauthier and Neil Gupta. We thank Hari lyer, Evariste Bigirimana and Jackline Odhiambo for their support in the data cleaning, analysis and manuscript editing. We also thank Janet Umuhoza and Liliose Mukantaganzwa for their help with data collection. We would like to acknowledge the Rwanda Ministry of Health for its leadership, and the 
doctors and nurses at Kirehe and Rwinkwavu District Hospitals who work tirelessly to improve the lives of newborns in their communities.

\section{Funding}

We acknowledge Partners In Health/Inshuti Mu Buzima and the IMB Innovation Grants for the support of this work. We acknowledge the PIH/IMB Doris Duke Charitable Foundation funds that supported data collection. The funding used for data collection for this study was part of a larger support by the Doris Duke Charitable Foundation. The funder had no direct role in any technical or intellectual aspect of this work.

\section{Availability of data and materials}

The data that support the findings of this study are available from Partners In Health/Inshuti Mu Buzima but restrictions apply to the availability of these data, which were used under license for the current study, and are not publicly available. Data are available from the corresponding author upon reasonable request and with permission of Partners In Health/Inshuti Mu Buzima and the Rwandan Ministry of Health.

\section{Authors' contributions}

MN led all aspects of the study from study design, data analysis and interpretation of results, and manuscript writing. BHG, NG and RB supported study design, analysis, and results interpretation and critically reviewed the manuscript. EN, WI, FN, FB, ChM, FM, CIM, ML and AH supported study design, and critically reviewed manuscript. HM supervised the study and supported design, results interpretation, and provided constructive feedback on the manuscript. All authors approved the final manuscript.

\section{Ethics approval and consent to participate}

The study received technical approval from the PIH/IMB Research Committee and the Rwanda National Health Research Committee and ethical approval from the Rwanda National Ethics Committee and the Institutional Review Board of Brigham and Women's Hospital, Boston, MA, USA. The study was completed using de-identified routinely collected program data; therefore, no informed consent was required.

\section{Consent for publication}

Not applicable.

\section{Competing interests}

The authors declare that they have no competing interests.

\section{Publisher's Note}

Springer Nature remains neutral with regard to jurisdictional claims in published maps and institutional affiliations.

\section{Author details}

${ }^{1}$ Partners In Health/Inshuti Mu Buzima (PIH/IMB), P.O. Box 3432, Kigali, Rwanda. ${ }^{2}$ Ministry of Health, Kigali, Rwanda. ${ }^{3}$ Department of Global Health and Social Medicine, Harvard Medical School, Boston, USA. ${ }^{4}$ Brigham and Women's Hospital, Boston, USA. ${ }^{5}$ Boston Children's Hospital, Boston, USA. Institute for Healthcare Improvement, Addis Ababa, Ethiopia.

Received: 22 November 2017 Accepted: 31 October 2018

Published online: 12 November 2018

\section{References}

1. Liu L, Oza S, Hogan D, Chu Y, Perin J, Zhu J, et al. Global, regional, and national causes of under-5 mortality in 2000-15: an updated systematic analysis with implications for the sustainable development goals. Lancet. 2016;388(10063):3027-35. https://doi.org/10.1016/S0140-6736(16)31593-8.

2. UNICEF. Every Child Alive: The urgent need to end newborn deaths. Genève: United Nation's Children's Fund, UNICEF; 2016. available at https:// www.unicef.org/publications/files/Every_Child_Alive_The_urgent_need_to_ end_newborn_deaths.pdf. Accessed 7 Nov 2018.

3. Rüegger C, Hegglin M, Adams M, Bucher HU. Population based trends in mortality, morbidity and treatment for very preterm- and very low birth weight neonates over 12 years. BMC Pediatr. 2010;12(1):17.

4. Kinney MV, Kerber KJ, Black RE, Cohen B, Nkrumah F, Coovadia H, et al. SubSaharan Africa's mothers, newborns, and children: where and why do they die? PLoS Med. 2010;7(6):e1000294.
5. Zaidi AK, Tikmani SS, Sultana S, Baloch B, Kazi M, Rehman H, Karimi K, et al Simplified antibiotic regimens for the management of clinically diagnosed severe infections in newborns and young facilities neonates in first-level in Karachi, Pakistan. Pediatr Infect Dis J. 2013;32(Suppl 1):S19-25. https://doi. org/10.1097/INF.0b013e31829ff7aa.

6. Martin S, Duke T, Davis P. Efficacy and safety of bubble CPAP in neonatal care in low and middle income countries: a systematic review. Arch Dis Child. 2014;99(6):F495-504

7. Aranda JV, Gorman W, Bergsteinsson H, Gunn T. Efficacy of caffeine in treatment of apnea in the low-birth-weight infant. J Pediatrics. 1977:90(3):467-72.

8. Schmidt B, Roberts RS, Davis P, Doyle LW, Barrington KJ, Ohlsson A, et al. Caffeine therapy for apnea of prematurity. N Engl J Med. 2006;354:2112-21. https://doi.org/10.1056/NEJMoa054065.

9. Bhutta ZA, Das JK, Bahl R, Lawn JE, Salam RA, Paul VK, et al. Can available interventions end preventable deaths in mothers, newborn babies, and stillbirths, and at what cost? Lancet. 2014;384(9940):347-70. https://doi.org/ 10.1016/S0140-6736(14)60792-3.

10. Darmstadt GL, Kinney MV, Chopra M, Cousens S, Kak L, Paul VK, Martines J, Bhutta ZA, Lawn JE. Who has been caring for the baby? Lancet. 2014; 384(9938):174-88. https://doi.org/10.1016/S0140-6736(14)60458.

11. Millennium Development Goals Rwanda Final Progress Report: 2013. Availabile at: http://www.rw.undp.org/content/rwanda/en/home/presscenter/articles/ 2015/03/31/millennium-development-goals-rwanda-final-progress-report-2013. html. Accessed 28 Aug 2016.

12. National Institute of Statistics of Rwanda. Rwanda Demographic and Health Survey 2014-2015: Final Report. Kigali: NISR; 2015. Available at: https:// dhsprogram.com/publications/publication-fr316-dhs-final-reports.cfm. Accessed 28 Aug 2016

13. Winter $R$, Thomas $P$, Langston A, Ndicunguye $V$, Mivumbi PC, Rutayisire DN, et al. Trends in neonatal mortality in Rwanda, 2000-2010. DHS Further Analysis Reports. Calverton, Maryland: ICF International; 2013. p. 88. Available at: http://www.dhsprogram.com/pubs/pdf/FA88/FA88.pdf. Accessed 7 Nov 2018

14. Farmer $P E$, Nutt $C T$, Wagner $C M$, et al. Reduced premature mortality in Rwanda: lessons from success. Br Med J. 2013;346:65 doi: 10/1136/bmj.f65.

15. Health Management Information Systems. National Institute of Statistics of Rwanda. Available at: http://statistics.gov.rw. Accessed 28 Aug 2016.

16. Kayinamura AM, Serubibi Y, Kakoma JB. Benefits of a neonatology service in a rural district hospital: case study of Rwamagana District hospital, Eastern Province of Rwanda. Rwanda Medical Journal. 2010;68:4.

17. Rwanda Ministry of Health. National neonatal care protocol. Kigali: MOH; 2012.

18. Hansen A, Magge $\mathrm{H}$, Labrecque M, Munyaneza RBM, Nahimana E, Nyishime $M$, et al. The development and implementation of a newborn medicine program in resource- limited setting. Public Health Action. 2015;5(1):17-22.

19. National Institute of Statistics of Rwanda. 2012 Population and Housing Census. Kigali: NISR; 2012. Available at: http://www.statistics.gov.rw/ publication/rphc4-atlas. Accessed 7 Nov 2018.

20. Hoque M, Kwadabeba CHC. Causes of neonatal admissions and deaths at a rural hospital in KwaZulu-Natal, South Africa. South Afr J Epidemiol Infect. 2011;26(1):26-9.

21. Ballot DE, Potterton J, Chirwa T, Hilburn N, Cooper PA. Developmental outcome of very low birth weight neonates in a developing country. BMC Pediatr. 2012;12(1):11.

22. Padayachee N, Ballot DE. Outcomes of neonates with perinatal asphyxia at a tertiary academic hospital in Johannesburg, South Africa. South Afr J Child Health. 2013;7(3):89-94.

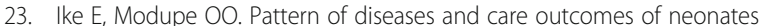
admitted in special care baby unit of university college hospital, Ibadan, Nigeria from 2007 to 2011. IOSR journal of nursing and health. Science. 2015:4(3):2320-1959.

24. Lawn JE, Blencowe $H$, Oza S, You D, Lee ACC, Waiswa P, Lalli M, Bhutta ZA, Barros AJD, Christian P, Mathers C, Cousens SN. Progress, priorities, and potential beyond survival. Lancet. 2014;384(9938):189-205.

25. Arcury TA, Gesler WM, Preisser JS, Sherman J, Spencer J, Perin J. The effects of geography and spatial behavior on health care utilization among the residents of a rural region. BMC Health Serv Res. 2005;40(1):135-55

26. Parkash J, Das N. Pattern of admissions to neonatal unit. J Coll Physicians Surg Pak. 2005;15(6):341-4

27. Seale AC, Blencowe $H$, Manu AA, Nair H, Bahl R, Oazi AS, et al. Estimates of possible severe bacterial infection in neonates in sub-Saharan Africa, South 
Asia, and Latin America for 2012: a systematic review and meta-analysis. Lancet Infect Dis. 2014;14:731-41.

28. Vergnano S, Sharland M, Kazembe P, Mwansambo C. Neonatal sepsis: an international perspective. Arch Dis Child. 2005;90:F220-4.

29. Holloway KA, Ivanovska V, Wagner AK, Vialle-Valentine C, Ross-Degnan D. Have we improved the used of medicines in developing and transitional countries and do we know how to? Two decades of evidence. Tropical Med Int Health. 2013;18(6):656-64. https://doi.org/10.1111/tmi.12123.

30. Bancalari E, Claure N. Advances in respiratory support for high risk newborn infants. Matern Health Neonatol Perinatol. 2015;1:13 doi.org/10.1186/s40748015-0014-5.

31. Chow S, Chow R, Popovic M, Lam M, Popovic M, Merrick J, Stashefsky M, Lam H, Milakovic M, Chow E, Popovic J. A selected review of the mortality rates of neonatal intensive care units. Front Public Health. 2015;3:225. https://doi.org/10.3389/fpubh.2015.00225.

32. Magge H, Manzi A, Rwabukwisi Cyamatare F, Mezzacappa C, Nkikabahizi F, Niyonzima S, Drobac PC, Ngabo F, Hirschhorn LR. Mentoring and quality improvement strengthen integrated management of childhood illness implementation in rural Rwanda. Arch Dis Child. 2014;0:1-6.

33. Manzi A, Magge H, Hedt-Gauthier BL, Michaelis AP, Cyamatare FR, Nyirazinyoye L, et al. Clinical mentorship to improve pediatric quality of care at the health centers in rural Rwanda: a qualitative study of perceptions and acceptability of health care workers. BMC Health Serv Res. 2014;14(1):275.

34. Nahimana E, Ngendahayo M, Magge H, Odhiambo J, Amoroso CL, Muhirwa E, Uwilingiyemungu JN, Nkikabahizi F, Habimana R, Hedt-Gauthier BL. Bubble CPAP to support preterm neonates in rural Rwanda: a retrospective cohort study. BMC Pediatr. 2015;15:135. https://doi.org/10.1186/s12887-015-0049-x.

35. Odhiambo J, Rwabukwisi FC, Rusangwa C, Rusanganwa V, Hirschhorn LR, Nahimana E, Ngamije P, Hedt-Gauthier BL. Health worker attrition at a rural district hospital in Rwanda: a need for improved placement and retention strategies. Pan Afr Med J. 2017;27:168.

36. Ingabire W, Reine PM, Hedt-Gauthier BL, Hirschhorn LR, Kirk CM, Nahimana E, Uwiringiyemungu JN, Ndayisaba A, Manzi A. Roadmap to an effective quality improvement and patient safety program implementation in a rural hospital setting. Healthcare. 2015;3:277-82. https://doi.org/10.1016/j.hjdsi. 2015.10.010

Ready to submit your research? Choose BMC and benefit from:

- fast, convenient online submission

- thorough peer review by experienced researchers in your field

- rapid publication on acceptance

- support for research data, including large and complex data types

- gold Open Access which fosters wider collaboration and increased citations

- maximum visibility for your research: over $100 \mathrm{M}$ website views per year

At $\mathrm{BMC}$, research is always in progress.

Learn more biomedcentral.com/submissions 